EPJ manuscript No.

(will be inserted by the editor)

\title{
The Localization Transition of the Two-Dimensional Lorentz Model
}

\author{
Teresa Bauer ${ }^{1}$, Felix Höfling ${ }^{2,3}$, Tobias Munk ${ }^{1}$, Erwin Frey ${ }^{1}$, and Thomas Franosch $^{4,1}$ \\ 1 Arnold Sommerfeld Center for Theoretical Physics (ASC) and Center for NanoScience (CeNS), \\ Fakultät für Physik, Ludwig-Maximilians-Universität München, Theresienstraße 37, 80333 München, \\ Germany \\ 2 Rudolf Peierls Centre for Theoretical Physics, 1 Keble Road, Oxford OX1 3NP, England, United \\ Kingdom \\ 3 Max Planck Institute for Metals Research, Heisenbergstraße 3, 70569 Stuttgart, Germany \\ 4 Institut für Theoretische Physik, Universität Erlangen-Nürnberg, Staudtstraße 7, 91058 Erlangen, \\ Germany
}

\begin{abstract}
We investigate the dynamics of a single tracer particle performing Brownian motion in a two-dimensional course of randomly distributed hard obstacles. At a certain critical obstacle density, the motion of the tracer becomes anomalous over many decades in time, which is rationalized in terms of an underlying percolation transition of the void space. In the vicinity of this critical density the dynamics follows the anomalous one up to a crossover time scale where the motion becomes either diffusive or localized. We analyze the scaling behavior of the time-dependent diffusion coefficient $D(t)$ including corrections to scaling. Away from the critical density, $D(t)$ exhibits universal hydrodynamic long-time tails both in the diffusive as well as in the localized phase.
\end{abstract}

\section{Introduction}

Understanding transport in heterogeneous media is fundamental for a variety of applications ranging from material sciences, porous catalysts, oil recovery, and even biological system. Often, the motion of particles inside such materials is strongly hindered due to the presence of slowly rearranging or immobilized obstacles of many different length scales. Many heterogeneous materials, e.g. rocks, soils, cements, foams and ceramics, consist of solid frames permeated by a network of pores [1, 2], and a mobile agent can meander through this static course of obstacles and display long-range transport. Likewise, transport in densely packed systems is strongly obstructed by the presence of surrounding particles via their excluded volume effect. In many cases a separation of time scales naturally occurs, for example in strongly heterogeneous mixtures such as sodium ions in silicates [3 - 5 ] or size-disparate soft or Yukawa spheres [6] 6 , leading to a much slower diffusion of one component. Similarly, the dense packing of differently sized proteins, lipids and sugars in the cell cytoplasm leads to strongly suppressed transport known as molecular crowding [9 12]. Again the motion of a smaller sized molecule is much faster than of surrounding macromolecules and the small molecule explores a quasi-static array of obstacles. Molecular crowding is also relevant in quasi two-dimensional systems such as protein diffusion in lipid bilayers as studied by single molecule fluorescence microscopy [13] or fluorescence correlation spectroscopy [14 16].

The motion of a tracer in these materials often displays anomalous transport as manifested in a subdiffusive increase of the mean-square displacement (MSD). This behavior is displayed in a finite window of time and a crossover to ordinary diffusion occurs at sufficiently long times. 
The exponent characterizing the subdiffusive behavior often appears to depend on the details of the system and even changes as the experimental parameters are varied.

The Lorentz model consisting of a single tracer exploring a course of randomly distributed frozen obstacles constitutes a minimal model for transport in heterogeneous media [17]. In its simplest variant the obstacles are spheres or disks and the positions are Poisson distributed. Hence, they may overlap and form clusters which restrict the motion of a pointlike tracer particle to the remaining void space. Above a certain obstacle density, the void space no longer permeates the entire system and a percolation transition occurs [18]. It has been shown that this transition is accompanied with a divergence of a characteristic length scale $\xi$, known as correlation length. The dynamics close to the transition displays critical behavior and the meansquare displacement exhibits behavior similar to experimental observations for heterogeneous media. It has been shown that the Lorentz model generically leads to large crossover windows explaining the apparent drift in characteristic exponents 19 21. Recently, the two-dimensional Lorentz model has been introduced in the context of lateral diffusion of proteins in the plasma membrane 22 24].

Systematic studies on the two-dimensional Lorentz model were mostly restricted to low densities focusing on the algebraic decay of the velocity autocorrelation functions (VACF) and the non-analytic dependence of the diffusion coefficient on the obstacle density [25 30]. The recurrent collisions with the obstacles lead to infinite memory resulting in a negative long-time tail $\sim-t^{-2}$ of the VACF 17, 31, 32]; yet close to the transition, the critical behavior shifts the onset of the hydrodynamic tail to longer and longer times [33]. The critical behavior of the Lorentz model in two-dimensions is expected to be qualitatively different from the threedimensional case since in the latter the conductances through narrow channels determines the dynamic exponent from pure geometric reasons [34, 35]. In the former the narrow gaps are expected to be less relevant and the universality of transport on a percolating lattice should be recovered. Beyond universality one would like to know the range of validity of the universal behavior, the size of the crossover region, and the importance of corrections to scaling.

In this work we present simulation results for the two-dimensional Lorentz model for Brownian tracer particles, in particular for densities close to the percolation transition. We have measured the mean-square displacement, the time-dependent diffusion coefficient, and the VACF, and analyze their respective critical behavior. Then we compare the subdiffusive behavior as well as the diffusion coefficient with the predicted power-law behavior. The non-algebraic decay of the VACF at long times emerges also for the case of a Brownian tracer corroborating the notion that the frozen configuration space alone gives rise to persistent correlations in the dynamics. A scaling theory that includes the leading corrections to scaling is developed and tested against the simulation data by suitable rectification plots.

\section{Lorentz Model}

The Lorentz model constitutes the minimal model for particle transport through a disordered material. In its simplest variant, a single classical tracer particle traverses a $d$-dimensional array of frozen hard-core obstacles of density $n$. Each obstacle acts as a scattering center of radius $\sigma$ restricting the motion of the tracer to the void space. For independently distributed scatterers the only control parameter characterizing the structure is then the dimensionless number density $n^{*}=n \sigma^{d}$. Equivalently, one may employ the porosity $\varphi$, i.e., the volume fraction accessible to the tracer due to the possibly overlapping obstacles. In the planar problem $(d=2)$ which we address in this work, one easily calculates

$$
\varphi=\exp \left(-\pi n \sigma^{2}\right)
$$

Already at intermediate obstacle density, the void space decomposes into many pockets of different sizes, and long-range particle transport occurs only through the void space that is percolating through the entire system. At a certain obstacle density $n_{c}^{*} \approx 0.359$, the infinite component ceases to exist and all particles are trapped in finite pockets. The problem of continuum percolation constitutes a critical phenomenon of purely geometric origin [18], and a 
series of predictions has been made for the characteristic behavior in the close vicinity of the critical density $n_{c}^{*}$. The linear dimension of the largest finite cluster (of the void space) defines the correlation length $\xi$, which is expected to diverge as $\xi \sim\left|n^{*}-n_{c}^{*}\right|^{-\nu}$ as the critical density is approached. Below the length scale $\xi$ the geometric structures appear, in a statistical sense, indistinguishable to the ones at $n_{c}^{*}$ forming the basis for the notion of self-similarity. Simultaneously, the root-mean-square size $\ell$ of all finite clusters diverges, yet with a smaller exponent $\ell \sim\left|n_{c}-n_{c}^{*}\right|^{-\nu+\beta / 2}$. The same exponent $\beta$ governs the vanishing of the relative weight of the infinite component as $n_{c}$ is increased towards the percolation threshold, $P_{\infty} \sim\left(n_{c}^{*}-n_{c}\right)^{\beta}$. With respect to geometric properties, continuum percolation shares the same universality class as lattice percolation, and in two dimensions the exact values of the exponents are known from a mapping to the Baxter line of the eight-vertex model $\nu=4 / 3, \beta=5 / 36$ [35 37$]$.

Transport of a single particle is expected to become anomalous and universal close to the percolation threshold independent of the details of the dynamics at the microscale. Here we consider a particle undergoing Brownian motion confined to the void space. Then the shorttime diffusion coefficient $D_{0}$ fixes the microscopic time scale $t_{0}:=\sigma^{2} / D_{0}$, i.e., the typical time needed for the particle to diffuse the distance of one obstacle radius without obstruction. The simplest quantity characterizing the motion of the tracer is the mean-square displacement $\delta r^{2}(t)=\left\langle[\mathbf{R}(t)-\mathbf{R}(0)]^{2}\right\rangle$, where the brackets indicate averaging both over all initial positions of the particle as well as different realizations of the disorder. In particular, particles that are initially in a finite pocket will remain there forever and do not contribute to long-range transport.

Directly at the percolation threshold $\left(n^{*}=n_{c}^{*}\right)$ the void space is self-similar and the dynamics of a walker becomes subdiffusive $\delta r^{2}(t) \sim t^{2 / z}$ for long times $t \gg t_{0}$. The dynamic exponent $z$ is independent of the geometric exponents of the percolation problem, but is determined from the universality class of random resistor networks. For obstacle densities above $n_{c}^{*}$, all particles are trapped in finite clusters and correspondingly the mean-square displacement is expected to saturate at the mean-square cluster size $\delta r^{2}(t \rightarrow \infty)=\ell^{2}$. However, close to the transition the subdiffusive behavior should be visible in a finite time window $t_{0} \ll t \ll t_{x}$ where $t_{x}$ denotes the crossover time to localization. These arguments suggest that for small separation parameter $\epsilon:=\left(n^{*}-n_{c}^{*}\right) / n_{c}^{*}$ the mean-square displacement should obey the scaling law

$$
\delta r^{2}(t ; \epsilon) \simeq t^{2 / z} \delta \hat{r}_{ \pm}^{2}(\hat{t}), \quad \hat{t}=t / t_{x}
$$

for $\epsilon \downarrow 0$ and $t \gg t_{0}$ and a scaling function $\delta \hat{r}_{+}^{2}(\cdot)$. We anticipate that scaling is also obeyed on the diffusive side $(\epsilon \uparrow 0)$ with a corresponding scaling function $\delta \hat{r}_{-}^{2}(\cdot)$ and the same crossover time scale $t_{x}$. To describe the crossover from critical dynamics to localization/diffusion, the scaling functions should exhibit the following asymptotics: $\delta \hat{r}_{ \pm}^{2}(\hat{t} \rightarrow 0)=$ const. and $\delta \hat{r}_{+}^{2}(\hat{t} \rightarrow$ $\infty) \sim \hat{t}^{-2 / z}, \delta \hat{r}_{-}^{2}(\hat{t} \rightarrow \infty) \sim \hat{t}^{1-2 / z}$, respectively. From the known long-time behavior on the localized side one infers for the crossover scaling time $t_{x} \sim \ell^{z} \sim|\epsilon|^{z(-\nu+\beta / 2)}$. Interestingly, the relevant length scale that determines the divergence of the inherited time is given by the mean cluster size $\ell$ rather than the correlation length $\xi$. Since $t_{x}$ also marks the crossover to the diffusive regime for $\epsilon<0$, one immediately concludes that the long-time diffusion coefficient should vanish as $D \sim(-\epsilon)^{\mu}$ for $\epsilon \uparrow 0$ with the conductivity exponent $\mu=(z-2)(\nu-\beta / 2)$. We use the value determined by Grassberger [38] in high-precision computer simulations for the electrical conductivity of a percolating lattice, $\mu=1.310 \pm 0.001$, as reference value and calculate the anomalous dimension to $z=3.036$.

\section{Simulation Results}

We have performed Brownian dynamics simulations for a single particle moving in a fixed array of hard disks of radius $\sigma$. The obstacles are distributed independently with a fixed average density $n$. We have employed periodic boundary conditions for system sizes of $L / \sigma=10,000$ and generated Brownian trajectories for very long times following an algorithm discussed in [39] which was also employed recently for the three-dimensional Lorentz model [20]. In essence, 


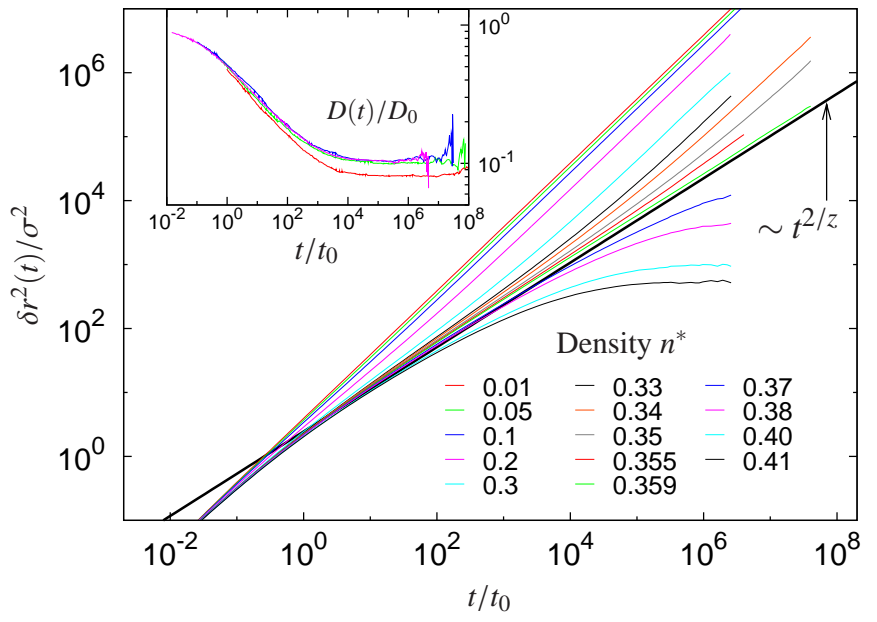

Fig. 1. Mean-square displacement $\delta r^{2}(t)$ of the Lorentz model for Brownian particles. The obstacle density $n^{*}$ increases from top to bottom; the thick black line indicates the longtime asymptote for anomalous transport at criticality $\delta r^{2}(t) \sim t^{2 / z}$ with $z=3.036$. The inset displays the timedependent diffusion coefficient $D(t)$ at obstacle density $n^{*}=0.30$ for varying algorithmic parameter $\tau_{B} / t_{0}=0.25$, $0.015625,0.0025$, and 0.0005625 .

particles are propagated along a deterministic, straight trajectory with specular scattering every time the tracer hits an obstacle, yet this dynamics is interrupted at regular time intervals $\tau_{B}$, where new velocities are drawn from a Maxwell distribution of variance $v^{2}$. Then on time scales longer compared to $\tau_{B}$ and length scales larger than $v \tau_{B}$, a free particle undergoes Brownian motion with diffusion coefficient $D_{0}=v^{2} \tau_{B} / 4$. In the presence of the obstacles the particle can still be considered as Brownian walker at the microscale with short-time diffusion coefficient $D_{0}$, provided $\tau_{B}$ is small relative to the inverse collision rate $\tau_{c}=1 / 2 \pi n \sigma v$. We shall use the characteristic time $t_{0}=\sigma^{2} / D_{0}$ as our basic unit of time, i.e., the time a free particle needs to traverse an obstacles radius. The algorithmic condition to mimic Brownian dynamics at the microscale is thus given by $\tau_{B} / t_{0} \ll 1 /\left(2 n^{*}\right)^{2}$. We have verified that $\tau_{B} / t_{0}=0.0025$ is sufficiently small in order that the long-time behavior is independent of the microparameters $v^{2}$ and $\tau_{B}$ as exemplified in the inset of Fig. 1 for a moderate obstacle density $n^{*}=0.3$. There the time-dependent diffusion coefficient is displayed for different $\tau_{B}$ and the curves neatly superimpose for $\tau_{B}<0.015625 t_{0}$. For the production runs we have fixed $\tau_{B}=0.0025 t_{0}$ and have calculated mean-square displacements as running-time averages over several trajectories for at least 100 different realizations of the disorder resulting in more than 775 trajectories in total for each density $n^{*}$ to generate high-accuracy data. The trajectories extend over huge time windows of typically $2.5 \times 10^{6} t_{0}$, yet close to the percolation threshold and for densities $n^{*} \leq 0.1515$ times longer trajectories have used. With current computing resources, a single trajectory at the critical density and for the longest simulation times runs approximately 40 hours on one core of a Quad Core Intel(R) Xeon(R) CPU X5365 (3.00 GHz).

Results for the mean-square displacement $\delta r^{2}(t)$ for all obstacle densities are exhibited in Fig. 1 on double logarithmic scales. First one should notice, that the data display almost no noise even for the longest times. For short times, all data start from the short-time diffusive motion, $\delta r^{2}(t)=4 D_{0} t$, and only at times $t \simeq t_{0}$ the presence of the obstacles suppresses the motion and the curves fan out. For low obstacle densities, the long-time behavior is again diffusive yet with a suppressed diffusion coeffficient $D\left(n^{*}\right)$. On the other hand, the mean-square displacements for high $n^{*}$ saturate at long times, reflecting the fact that all particles are localized.

The localized and diffusive curves are nicely discriminated by a critical density $n_{c}^{*}=0.359$ where the MSD behaves subdiffusively over at least six decades in time, i.e., it extends to our longest observation times. This critical density coincides with the numerical estimate $n_{c}^{*}=0.359072$ (4) for continuum percolation [40, 41]. The value $z=3.036$, as inferred from the exponent $\mu$ determined by finite-size scaling of the conductivity at the critical point [38] provides an excellent description of the long-time behavior of the critical MSD $\delta r^{2}(t) \sim t^{2 / z}$. Our simulations provide the first quantitative test that the $2 \mathrm{~d}$ Lorentz model shares the universality class of two-dimensional random resistor networks. 


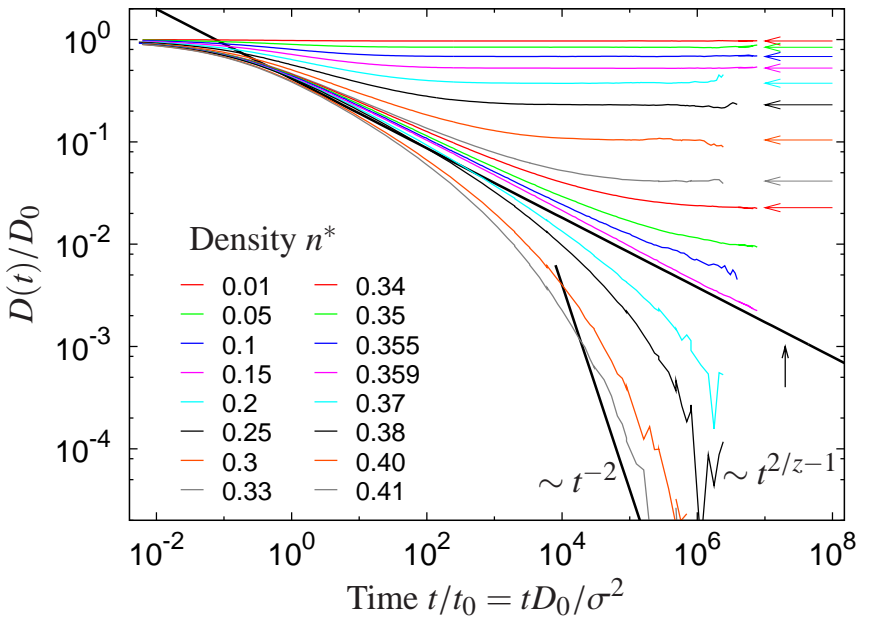

Fig. 2. The time-dependent diffusion coefficient $D(t):=(1 / 4) \mathrm{d} \delta r^{2}(t) / \mathrm{d} t$. The obstacle density $n^{*}$ increases from top to bottom; the arrows indicate the long-time diffusion coefficient $D$. For the critical density, the timedependent diffusion coefficient vanishes as a power law, $D(t) \sim t^{2 / z-1}$. The thick line indicates the power law $t^{-2}$ expected as long-time asymptote in the localized regime due to the culde-sacs.

For densities close to $n_{c}^{*}$, the data follow the critical one up to some finite time where they cross over to either diffusive or localized behavior. By naked eye one infers already that this crossover time increases as the critical density is gradually approached. Let us mention that in the three-dimensional Lorentz model, the curves off the critical point deviate much more from the critical one, than in the planar Lorentz model. Nevertheless they still display subdiffusion in a finite time window, yet with apparent density-dependent exponents [19, 20].

\subsection{Time-dependent diffusion coefficients}

A quantity more sensitive to the anomalous transport behavior is given in terms of the timedependent diffusion coefficient

$$
D(t):=\frac{1}{2 d} \frac{\mathrm{d}}{\mathrm{d} t} \delta r^{2}(t)
$$

where the dimension is $d=2$ for the planar problem. We have taken numerical derivatives of the MSDs taking advantage of the fact that $\delta r^{2}(t)$ varies significantly only on logarithmic scales. Since the MSDs are calculated using our standard blocking scheme [42], the numerical derivatives essentially do not introduce new noise to the data. The time-dependent diffusion coefficient $D(t)$ is displayed in Fig. 2 for the same densities considered above. First, one notices that all curves start from the short-time diffusion constant $D_{0}$, corroborating that our numerical algorithm reproduces Brownian motion at small time and length scales. For increasing time $D(t)$ is gradually suppressed reflecting that obstacles can only slow down the overdamped dynamics. For densities below $n_{c}^{*}$ the time-dependent diffusion coefficient approaches a nonzero limit $D$ for long-times. The values of the long-time diffusion constant $D$ decrease rapidly as the critical density is approached from below. Directly at the critical point, $D(t)$ reaches a power-law long-time asymptote $D(t) \sim t^{2 / z-1}$ corresponding to a subdiffusive mean-square displacement. For densities above the critical one, the time-dependent diffusion coefficient vanishes even more rapidly. Following the argument of persistent correlations due to power-law distributed exit rates of the cul-de-sacs, one should expect a universal long-time tail $D(t) \sim t^{-2}$ in the entire localized phase [34]. Such a behavior has indeed been observed recently in molecular dynamics simulations for the two-dimensional Lorentz model for ballistic particles [33], though they considered the velocity autocorrelation function rather than $D(t)$. Our simulations exhibit clear evidence that this tail remains present for Brownian particles too as we shall argue below.

The diffusion constants $D$ extracted as long-time limits of $D(t)$ are displayed in Fig. 3 for varying obstacle density. Over the investigated range of densities, the diffusion constant is suppressed by a factor of 100. It vanishes as the critical density is approached and follows amazingly well the scaling prediction $D \sim(-\epsilon)^{\mu}$. Even for the lowest density considered, where the motion is practically unobstructed by the obstacles, $D\left(n^{*}=0.01\right)=0.97 D_{0}$ deviates by only 


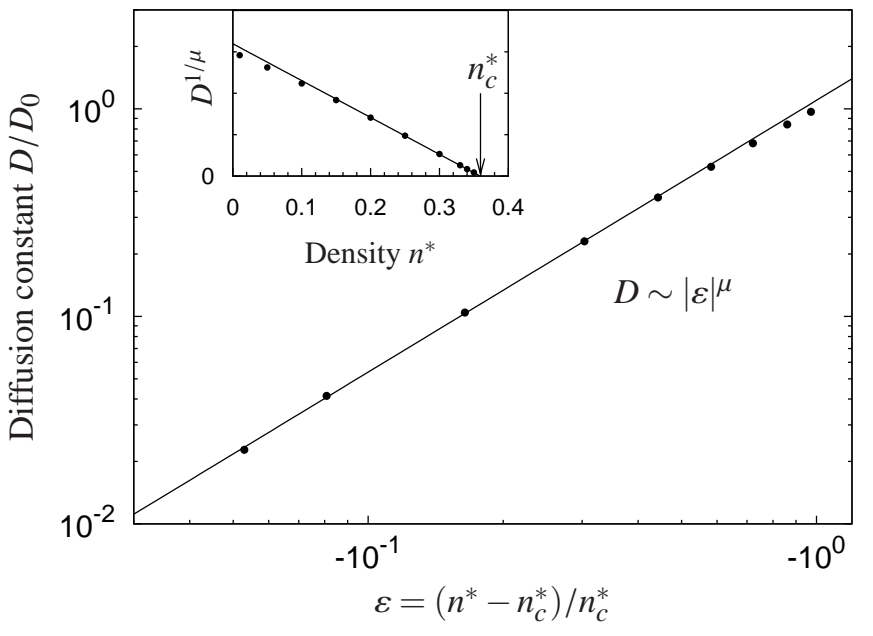

Fig. 3. Scaling behavior of the longtime diffusion coefficient $D$ with separation parameter $\epsilon=\left(n^{*}-n_{c}^{*}\right) / n_{c}^{*}$. The straight line in the rectification plot (inset) confirms the value of the conductivity exponent $\mu=1.309$.

$8.4 \%$ from the scaling asymptote. It appears as a coincidence that the critical regime connects down to the low-density asymptote without an intermediate region of moderate obstructed motion. For the corresponding three-dimensional system the convergence towards the scaling behavior is approached slowlier, however, since the corresponding conductivity exponent is much higher $\mu_{3 \mathrm{~d}}=2.88$, the diffusion vanishes much more rapidly and a suppression by five orders of magnitude can be observed [20]. The rectification plot in the inset of Fig. 3 corroborates that $\mu=1.310$ obtained by measuring the conductivity on a lattice close to percolation is indeed the correct value. Our simulations for Brownian particles provide an independent test that two-dimensional random resistor networks and the planar Lorentz model indeed share the same universality class. The critical density has been determined by extrapolating the straight line in the rectification plot to zero diffusivity, yielding $n_{c}^{*}=0.359 \pm 0.001$. The thus determined value of $n_{c}^{*}$ was used throughout this work to simulate the critical dynamics.

\subsection{Velocity autocorrelation functions}

Let us also discuss the velocity autocorrelation function (VACF), $Z(t)=\langle\mathbf{v}(t) \cdot \mathbf{v}(0)\rangle / d$, for the Brownian particle. Although the notion of velocity for Brownian particles is conceptually questionable, their correlation function is well defined for times $t>0$. Here we rely again on numerical derivatives, i.e., we employ

$$
Z(t):=\frac{1}{2 d} \frac{\mathrm{d}^{2}}{\mathrm{~d} t^{2}} \delta r^{2}(t)
$$

as definition. Then the relation to the time-dependent diffusion coefficient is provided by

$$
D(t)=D_{0}+\int_{0^{+}}^{t} Z\left(t^{\prime}\right) \mathrm{d} t^{\prime},
$$

where the integral is evaluated excluding the time $t=0$. This form constitutes the analog of the Green-Kubo relation, alternatively one can include a $\delta$-distribution in the VACF to account for the Brownian motion at the microscale.

Figure 4 displays the VACF, and one first observes that it is negative for all times, except on time scales associated with our algorithmic microparameter $\tau_{B}$. This fact is consistent with the notion that obstacles can only slow down the diffusion, Eq. (5), and in the case of Brownian motion one can show that the VACF is a completely monotone function, see Appendix A. The long-time behavior for the diffusive regime $\left(n<n_{c}\right)$ is characterized by persistent correlations that slowly decay as a power-law. The low densities display a tail $Z(t) \sim-t^{-2}$ consistent 

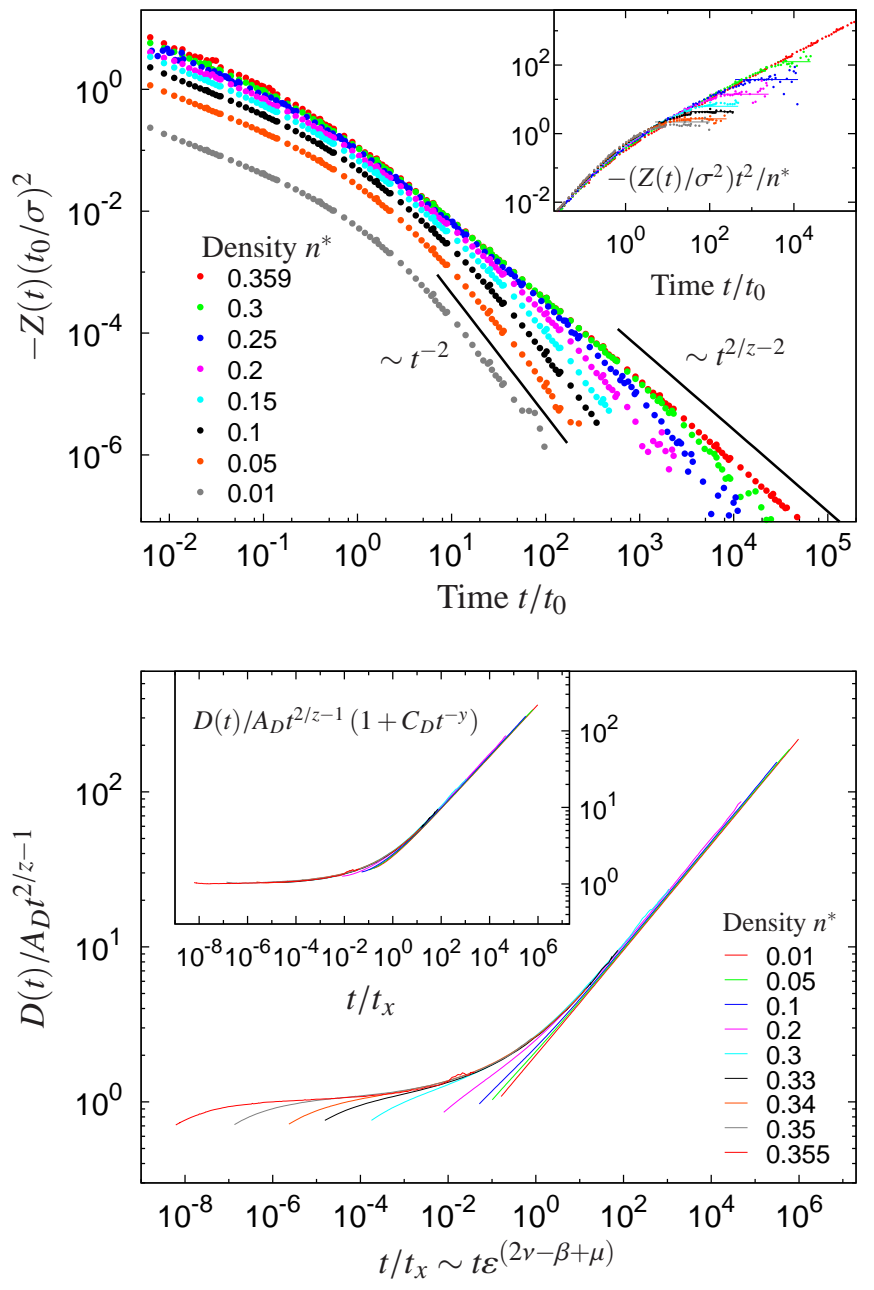

Fig. 4. Velocity autocorrelation function $Z(t)=(1 / 4) \mathrm{d}^{2} \delta r^{2}(t) / \mathrm{d} t^{2}$ for Brownian particles in the planar Lorentz model. The thick lines indicate the hydrodynamic tail $t^{-2}$ and the critical behavior $t^{2 / z-2}$, respectively. The inset displays a rectification plot $-t^{2} Z(t) / \sigma^{2} / n^{*}$ as a function of time.

Fig. 5. Scaling behavior of the timedependent diffusion coefficient for the densities below $n_{c}^{*}$. The scaling variable reads $t_{x}=t_{0}|\epsilon|^{\beta-2 \nu-\mu}$ The inset includes the leading correction to scaling with a correction amplitude $C_{D}=-0.14 t_{0}^{y}$ consistent with the critical density.

with the theoretical prediction for kinetic theory for ballistic particles [32]. It has been anticipated earlier [17] that also Brownian particles exhibit the same behavior, since the long-time correlations originate from repeated encounters of the same frozen heterogeneities. Indeed the Lorentz model for Brownian tracers can be solved analytically to lowest order in the scattering density $n^{*}$ and the time-dependence of the VACF including its long-time tail can be worked out exactly [43]. Nevertheless, to the best of our knowledge, our simulation results provide the first direct evidence for this universality at all densities. As the density is gradually increased, the overall signal in the VACF becomes larger and the exponent of the power-law tail appears to drift. A rectification plot shows that the $t^{-2}$ behavior is assumed for all densities as the late-time relaxation. The critical asymptote appears in an intermediate window which extends to longer and longer times as the critical density is approached. Our data also show that the amplitude of the hydrodynamic tail diverges close to $n_{c}^{*}$ which can be rationalized using the same arguments as in the ballistic case [33].

\section{Dynamic scaling analysis}

The power-law behavior in both the mean-square displacement or the time-dependent diffusion coefficient at criticality and the vanishing of the diffusion constant as a power law upon approaching the critical density is merely one aspect of the critical behavior. Yet, the universality 


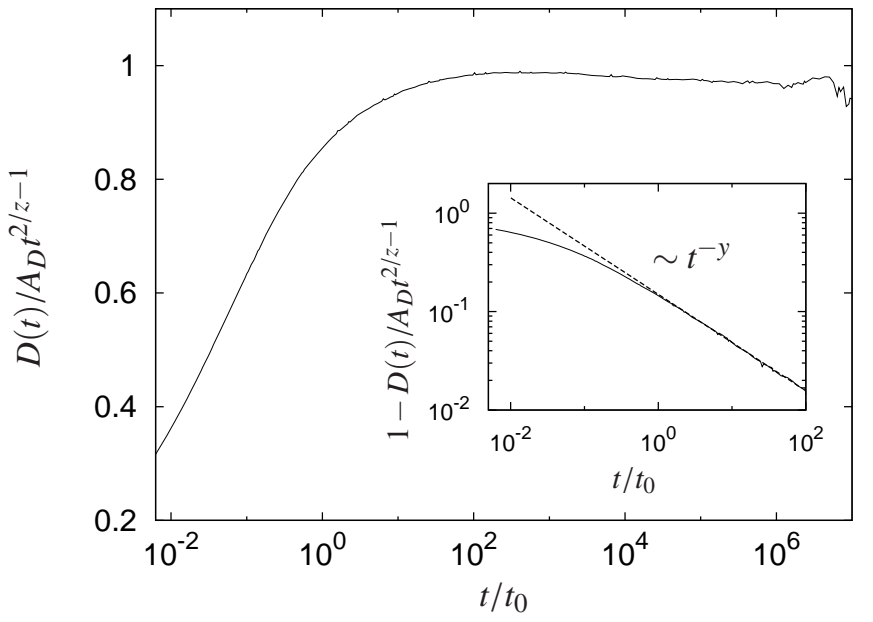

Fig. 6. Rectification of the timedependent diffusion coefficient $D(t)$ at the critical density $n_{c}^{*}$. The critical amplitude is determined to $A_{D}=$ $0.508 D_{0} t_{0}^{1-2 / z}$. The inset displays the approach of the MSD towards the critical law. The thick line indicates a power laws with the universal correction exponent $y=0.49$.

hypothesis suggests a much more sensitive test in terms of scaling. For example, the meansquare displacement $\delta r^{2}(t ; \epsilon)$ for small separation parameters $|\epsilon| \ll 1$ and long times $t \gg t_{0}$ is expected to fulfill Eq. (2). Here we show that the time-dependent diffusion coefficient $D(t ; \epsilon)$ can be used equivalently to test the scaling prediction. Taking derivatives, we suggest

$$
D(t ; \epsilon) \simeq t^{2 / z-1} \hat{D}_{ \pm}(\hat{t}), \quad \hat{t}=t / t_{x}
$$

with the scaling time $t_{x}:=t_{0}|\epsilon|^{-(2 \nu-\beta+\mu)}$. The connection with the scaling function for the mean-square displacement is easily established,

$$
\hat{D}_{ \pm}(\hat{t})=\frac{1}{2 d}\left[\frac{2}{z} \delta \hat{r}_{ \pm}^{2}(\hat{t})+\hat{t} \frac{\mathrm{d}}{\mathrm{d} \hat{t}} \delta \hat{r}^{2}(\hat{t})\right] .
$$

For short rescaled times, $\hat{D}_{ \pm}(\hat{t} \rightarrow 0)=$ const. $=: A_{D}$ and the critical behavior is recovered. For long times $\hat{D}_{-}(\hat{t}) \sim \hat{t}^{1-2 / z}$ such that diffusion is reached for $\epsilon<0$. On the localized side, the mean-square displacements saturate and the leading behavior $\delta r_{+}^{2}(\hat{t}) \sim \hat{t}^{-2 / z}$ cancels exactly in Eq. 7 thus $\hat{D}_{+}(\hat{t})=o\left(\hat{t}^{-2 / z}\right)$.

Figure 5 displays a rectification plot for $D(t)$ for obstacle densities below the critical ones. For large rescaled times the curves nicely superimpose, though this reflects merely the fact that the diffusion regime is reached for all cases and that the long-time diffusion coefficient obeys the scaling prediction $D \sim(-\epsilon)^{\mu}$. For short rescaled times the curves converge to a constant which is given by the long-time behavior of the time-dependent diffusion coefficient at the critical point. The fanning out of the curves arises due to corrections to scaling and eventually because of the crossover to the microscopic regime.

To gain further insight into the scaling behavior we extend our scaling hypothesis by a generic irrelevant scaling variable. Then it has been shown recently within a cluster-resolved scaling theory [4] that the mean-square displacement should obey

$$
\delta r^{2}(t ; \epsilon)=t^{2 / z} \delta \hat{r}_{ \pm}^{2}(\hat{t})\left[1+t^{-y} \Delta_{ \pm}(\hat{t})\right],
$$

where $y$ is another universal exponent characterizing the approach of the critical dynamical behavior. It is connected to a correction-to-scaling exponent $\Omega$ for the cluster-size distribution via the scaling relation $y=\Omega(\nu d-\beta) /[z(\nu-\beta / 2)]$. For the two-dimensional case the value $y=0.49(3)$ [4] was determined for random walks on a lattice, which we shall use in the following. Taking derivatives with respect to time, the corresponding prediction for the timedependent diffusion coefficient is

$$
D(t ; \epsilon) \simeq t^{2 / z-1} \hat{D}_{ \pm}(\hat{t})\left[1+t^{-y} \Delta_{ \pm}^{D}(\hat{t})\right],
$$


where the new scaling function $\Delta_{ \pm}^{D}(\hat{t})$ is connected to the one of the mean-square displacement via

$$
\begin{aligned}
2 d \hat{D}_{ \pm}(\hat{t}) \Delta_{ \pm}^{D}(\hat{t})= & \left(\frac{2}{z}-y\right) \delta \hat{r}_{ \pm}^{2}(\hat{t}) \Delta_{ \pm}(\hat{t}) \\
& +\hat{t} \frac{\mathrm{d}}{\mathrm{d} \hat{t}}\left[\delta \hat{r}_{ \pm}^{2}(\hat{t}) \Delta_{ \pm}(\hat{t})\right] .
\end{aligned}
$$

For small rescaled times the correction-to-scaling function reduces to a constant $\Delta_{ \pm}^{D}(\hat{t} \rightarrow 0)=$ const. $=: C_{D}$. and one easily infers the relation to the correction-to-scaling constant $C:=$ $\Delta_{ \pm}(\hat{t} \rightarrow 0)$ for the mean-square displacement: $C_{D}=C(2-y z) / 2$. In particular at criticality, the time-dependent diffusion coefficient displays a power-law correction for long times

$$
D(t ; \epsilon=0) \simeq A_{D} t^{2 / z-1}\left[1+C_{D} t^{-y}\right] .
$$

For long rescaled times $\hat{t} \rightarrow \infty$, the correction to scaling function behaves as a power-law again $\Delta_{-}^{D}(\hat{t} \rightarrow \infty) \simeq \tilde{C}_{ \pm}^{D} \hat{t}^{y}$, yielding corrections for the asymptotic behavior of the long-time diffusion constant

$$
D(\epsilon) \sim(-\epsilon)^{\mu}\left[1+\tilde{C}_{-}^{D} t_{0}^{-y}(-\epsilon)^{y \mu z /(z-2)}\right] .
$$

The time-dependent diffusion coefficient $D(t)$ at the critical density is displayed in Fig. 6 in a rectification plot. Within the statistical errors of our simulation one observes a saturation at long times provided the established value for $z$ is used, indicating that the asymptotic behavior is reached. At very long times the curve starts to deviate again due to statistical fluctuations, possibly finite-size effects, and the uncertainty of the value for the critical obstacle density. The amplitude of the critical relaxation $A_{D}=\lim _{t \rightarrow \infty} t^{1-2 / z} D(t ; \epsilon=0)$ has been determined to $A_{D}=0.508 D_{0} t_{0}^{1-2 / z}$ from our numerical data to optimize data collapse for the scaling in the diffusive and localized regime, see below. The approach towards this power-law behavior is consistent with a power law according to Eq. (11) with a correction to scaling amplitude $C_{D}=-0.14 t_{0}^{y}$, see inset of Fig. 6.

It appears that for a deeper analysis of the correction to scaling behavior as suggested by Eqs. (8) and (9), the precise form of the scaling function $\Delta_{ \pm}^{D}(\hat{t})$ has to be known. From general arguments, we know that the correction function for the MSD, $\Delta_{ \pm}(\hat{t})$, smoothly interpolates between a constant $\Delta_{ \pm}(\hat{t} \rightarrow 0)=: C$ and power laws $\Delta_{ \pm}(\hat{t} \rightarrow \infty) \simeq \tilde{C}_{ \pm} \hat{t}^{y}$. Then the correction term for the time-dependent diffusion coefficient behaves for large rescaled times $\hat{t} \rightarrow \infty$ as $\hat{D}(\hat{t}) \Delta_{-}^{D}(\hat{t}) \sim \hat{t}^{y-2 / z}$ on the diffusive side and $\hat{D}(\hat{t}) \Delta_{-}^{D}(\hat{t})=o\left(\hat{t}^{y-2 / z}\right)$ in the localized regime. Upon closer inspection of the correction behavior, Eq. (12), and the simulation results, Fig. [3. the corrections are very small for large rescaled times. Then one may approximate $\Delta_{ \pm}^{D}(\hat{t})$ by its short-time asymptote $\Delta_{ \pm}^{D}(\hat{t})=C_{D}$ for all rescaled times, i.e., ignore the corrections on the diffusive/localized time regime. The result of this procedure is displayed in the inset of Fig. 5 for the diffusive side with a quite impressive improvement of the scaling behavior. Let us emphasize that the only new parameter $C_{D}$ is in principle fixed by the critical behavior, such that no free parameters enter the scaling plot.

The scaling behavior for the time-dependent diffusion coefficient $D(t)$ is tested for the localized regime, $n^{*}>n_{c}^{*}$, in Fig. [7. Again the curves approach a constant at short rescaled times as the critical density is approached. Accounting for the correction by the same procedure as for the diffusive side yields an almost perfect data collapse without any new parameters. The scaling behavior at large times reflects that also the approach of the saturation in the MSDs is universal. From our general discussion, the scaling function is known to vanish rapidly $\hat{D}_{+}(\hat{t})=o\left(\hat{t}^{-2 / z}\right)$. Following Machta and Moore 34] there should universal power-law correlations in the entire localized phase, $D(t) \sim t^{-2}$ for $t \rightarrow \infty$, due to the meandering of the particle in the self-similar cul-de-sacs. Assuming that the crossover from the critical law to these universal hydrodynamic tails is again given by $t_{x}$ as we have argued earlier for ballistic particles [33], leads to a scaling prediction of $\hat{D}_{+}(\hat{t}) \sim \hat{t}^{-2 / z-1}$. In particular, one expects a divergence of the prefactor of the tail in the localized phase according to $t_{x}^{2 / z+1}$. This prediction is indicated in Fig. 7 and provides a nice description of the data. 


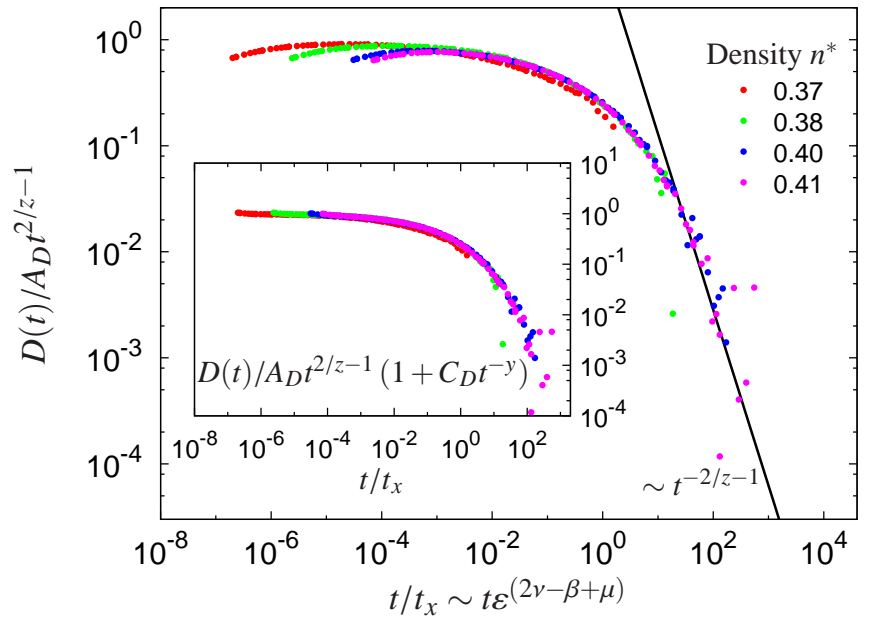

Fig. 7. Scaling behavior of the timedependent diffusion coefficient for the densities above $n_{c}^{*}$ with the crossover time $t_{x}=t_{0}|\epsilon|^{\beta-2 \nu-\mu}$. The thick line indicates the power law $t^{-2 / z-1}$ which is expected to hold in the entire localized phase. The inset includes the leading correction to scaling with the same correction amplitude $C_{D}=$ $-0.14 t_{0}^{y}$ as in the diffusive regime.

\section{Conclusion}

The dynamics of a tracer particle in a densely packed planar course of obstacles has been investigated by Brownian dynamics simulation. The slowing down of the dynamics close to the percolation threshold is accompanied by critical behavior observed over more that 6 decades in time. We corroborate that the planar Lorentz model shares a universality class with the random resistor network where the critical exponents are known from earlier simulations. We have shown that the time-dependent diffusion coefficient constitutes a suitable quantity to analyze the scaling behavior close to the transition. The corresponding scaling relations have been derived and extended by the leading correction. We find that scaling behavior is in general well obeyed and the corrections to scaling appear much less important than for the three-dimensional case [20].

The Lorentz model exhibits power-law long-time anomalies away from the critical density due to repeated encounters with the same scatterer. These tails have been derived originally for ballistic particles, yet they turn out to be universal irrespective of the dynamics at microscopic scales. Then the velocity autocorrelation, defined via a second derivative of the mean-square displacement exhibits the tails even for Brownian tracers. On the localized side we also find long-time tails due to the self-similar distribution of exit times of the cul-de-sacs [34], again irrespective of the microscopic dynamics. Interestingly, these long-time tails are part of the scaling function for the time-dependent diffusion coefficient.

The assumption that the obstacles are distributed independently is certainly an oversimplification in real systems. Then one would like to extend the Lorentz model where the matrix consists of some frozen-in configuration of a strongly interacting liquid or a snapshot of a slowly rearranging matrix of obstacles. Second, experiments are usually for a finite concentration of particles meandering in the array of obstacles and one may ask at what time and length scales these interaction of the tracers modifies the dynamics in the labyrinth. In three dimensions an intriguing interplay of the physics of the glassy dynamics and the localization transition has been discovered recently [3 8 ], and since the glass transition in two-dimensions is qualitatively similar [45, 46] one may hope that the physics of the planar Lorentz model is applicable in size-disparate two-dimensional mixtures.

Financial support from the Deutsche Forschungsgemeinschaft via contract No. FR 850/6-1 and from the Konrad-Adenauer-Stiftung (T.B.) is gratefully acknowledged. This project is supported by the German Excellence Initiative via the program "Nanosystems Initiative Munich (NIM)." 


\section{A Appendix: Completely monotone functions}

In this Appendix we develop a spectral representation for the mean-square displacement and the velocity-autocorrelation function for arbitrary dimension $d$.

For a Brownian particle in an external potential $U(\mathbf{r})$ the time-evolution of the conditional probability distribution $\Psi(\mathbf{r}, t)$ to find the particle at $\mathbf{r}$ at time $t$ provided it has been at $\mathbf{r}^{\prime}$ and some earlier time $t^{\prime}$ is governed by the Smoluchowski equation

$$
\partial_{t} \Psi\left(\mathbf{r}, t \mid \mathbf{r}^{\prime} t^{\prime}\right)=\frac{\partial}{\partial \mathbf{r}}\left[\frac{D_{0}}{k_{B} T} \frac{\partial U}{\partial \mathbf{r}} \Psi\right]+D_{0} \frac{\partial^{2} \Psi}{\partial \mathbf{r}^{2}} \equiv \hat{\Omega}(\mathbf{r}) \Psi
$$

where $\hat{\Omega}(\mathbf{r})$ denotes the Smoluchowski operator acting on the position $\mathbf{r}$. At the very end, we are interested in hard potentials with infinite barriers, however we anticipate that this case is assumed as limit of smooth potentials becoming increasingly steep.

One can also consider the evolution of $\Psi$ with respect to the conditional time and one can show that

$$
-\partial_{t^{\prime}} \Psi\left(\mathbf{r}, t \mid \mathbf{r}^{\prime} t^{\prime}\right)=-\frac{D_{0}}{k_{B} T} \frac{\partial U}{\partial \mathbf{r}^{\prime}} \frac{\partial \Psi}{\partial \mathbf{r}^{\prime}}+D_{0} \frac{\partial^{2} \Psi}{\partial \mathbf{r}^{\prime 2}} \equiv \hat{\Omega}^{+}\left(\mathbf{r}^{\prime}\right) \Psi,
$$

where the adjoint $\hat{\Omega}^{+}\left(\mathbf{r}^{\prime}\right)$ is with respect to the standard scalar product. Furthermore, $\hat{\Omega}^{+}\left(\mathbf{r}^{\prime}\right)$ is identified with the backward Smoluchowski operator and now acts on $\mathbf{r}^{\prime}$.

The mean-square displacement is obtained as an average

$$
\delta r^{2}\left(t-t^{\prime}\right) \equiv \int \mathrm{d} \mathbf{r} \mathrm{d} \mathbf{r}^{\prime}\left(\mathbf{r}-\mathbf{r}^{\prime}\right)^{2} \Psi\left(\mathbf{r} t \mid \mathbf{r}^{\prime} t^{\prime}\right) \Psi_{\mathrm{eq}}\left(\mathbf{r}^{\prime}\right),
$$

where $\Psi_{\text {eq }}(\mathbf{r})=Z^{-1} \exp \left(-U(\mathbf{r}) / k_{B} T\right)$ denotes the equilibrium distribution. In principle one may also introduce a disorder average for different realizations of the potential $U(\mathbf{r})$, yet we anticipate that for large enough systems the quantities of interest are self-averaging. Since in equilibrium the MSD is stationary, it depends only on the time difference and one derives

$$
\begin{aligned}
& \frac{\mathrm{d}^{2}}{\mathrm{~d} t^{2}} \delta r^{2}\left(t-t^{\prime}\right)=-\frac{\mathrm{d}}{\mathrm{d} t} \frac{\mathrm{d}}{\mathrm{d} t^{\prime}} \delta r^{2}\left(t-t^{\prime}\right) \\
& =\int \mathrm{d} \mathbf{r} \mathrm{d} \mathbf{r}^{\prime}\left(\mathbf{r}-\mathbf{r}^{\prime}\right)^{2}\left[\hat{\Omega}(\mathbf{r}) \hat{\Omega}^{+}\left(\mathbf{r}^{\prime}\right) \Psi\left(\mathbf{r} t \mid \mathbf{r}^{\prime} t^{\prime}\right)\right] \Psi_{\mathrm{eq}}\left(\mathbf{r}^{\prime}\right) \\
& =\int \mathrm{d} \mathbf{r} \mathrm{d} \mathbf{r}^{\prime} \Psi\left(\mathbf{r} t \mid \mathbf{r}^{\prime} t^{\prime}\right)\left[\hat{\Omega}\left(\mathbf{r}^{\prime}\right) \hat{\Omega}^{+}(\mathbf{r})\left(\mathbf{r}-\mathbf{r}^{\prime}\right)^{2} \Psi_{\mathrm{eq}}\left(\mathbf{r}^{\prime}\right)\right] \\
& =\int \mathrm{d} \mathbf{r} \mathrm{d} \mathbf{r}^{\prime} \Psi\left(\mathbf{r} t \mid \mathbf{r}^{\prime} t^{\prime}\right)\left[\hat{\Omega}^{+}\left(\mathbf{r}^{\prime}\right) \hat{\Omega}^{+}(\mathbf{r})\left(\mathbf{r}-\mathbf{r}^{\prime}\right)^{2}\right] \Psi_{\mathrm{eq}}\left(\mathbf{r}^{\prime}\right) \\
& =-2 \int \mathrm{d} \mathbf{r} \mathrm{d} \mathbf{r}^{\prime}\left[\hat{\Omega}^{+}(\mathbf{r}) \mathbf{r}\right] \cdot\left[\hat{\Omega}^{+}\left(\mathbf{r}^{\prime}\right) \mathbf{r}^{\prime}\right] \Psi\left(\mathbf{r} t \mid \mathbf{r}^{\prime} t^{\prime}\right) \Psi_{\mathrm{eq}}\left(\mathbf{r}^{\prime}\right),
\end{aligned}
$$

where in the second to last line the property of the Smoluchowski operator $\hat{\Omega}(\mathbf{r})\left[A(\mathbf{r}) \Psi_{\text {eq }}(\mathbf{r})\right]=$ $\left[\hat{\Omega}^{+}(\mathbf{r}) A(\mathbf{r})\right] \Psi_{\text {eq }}(\mathbf{r})$ valid for any well-behaved function $A(\mathbf{r})$ has been employed. The preceding result shows that the second derivative of the MSD can be interpreted essentially as the negative of the autocorrelation function of $\hat{\Omega}^{+}(\mathbf{r}) \mathbf{r}$. To make connection with the ballistic case it is helpful to introduce $\mathbf{v}=\mathrm{i} \hat{\Omega}^{+}(\mathbf{r}) \mathbf{r}$ as a formal velocity, and one recovers the usual relation to the velocity autocorrelation function

$$
\left\langle\mathbf{v}(t) \cdot \mathbf{v}\left(t^{\prime}\right)\right\rangle=\frac{1}{2} \frac{\mathrm{d}^{2}}{\mathrm{~d} t^{2}} \delta r^{2}\left(t-t^{\prime}\right)
$$

Next we recall that autocorrelation functions $C(t)=\left\langle A(t)^{*} A(0)\right\rangle$ for overdamped dynamics are completely monotone, i.e., their derivatives exhibit fixed sign

$$
(-1)^{n} \frac{\mathrm{d}^{n}}{\mathrm{~d} t^{n}} C(t) \geq 0 \quad \text { for all } n \in \mathbb{N}_{0}, \quad t \geq 0 .
$$


A sketch of a non-rigorous proof is as follows. First, consider the complex scalar product

$$
\langle A \mid B\rangle=\int \mathrm{d} \mathbf{r} A(\mathbf{r})^{*} B(\mathbf{r}) \Psi_{\mathrm{eq}}(\mathbf{r})
$$

Then one easily verifies that $\hat{\Omega}^{+}$is hermitian with respect to this scalar product $\left\langle A \mid \Omega^{+} B\right\rangle=$ $\left\langle\Omega^{+} A \mid B\right\rangle$. Then with the formal solution of Eq. (13), $\Psi\left(\mathbf{r} t \mid \mathbf{r}^{\prime} t^{\prime}\right)=\exp \left[\left(t-t^{\prime}\right) \hat{\Omega}(\mathbf{r})\right] \delta\left(\mathbf{r}-\mathbf{r}^{\prime}\right)$, one finds a representation of the autocorrelation function as

$$
C(t)=\left\langle\mathrm{e}^{\Omega^{+} t} A \mid A\right\rangle .
$$

Yet since $\hat{\Omega}^{+}$is hermitian all eigenvalues are real, and by a 'variational principle',

$$
\left\langle\hat{\Omega}^{+} A \mid A\right\rangle=-D_{0} \int \mathrm{d} \mathbf{r}\left|\frac{\partial A(\mathbf{r})}{\partial \mathbf{r}}\right|^{2} \Psi_{\mathrm{eq}}(\mathbf{r}) \leq 0
$$

also negative semi-definite. Zero constitutes the non-degenerate eigenvalue with constant eigenfunction $|0\rangle$. A spectral decomposition of the backwards Smoluchowski operator $\hat{\Omega}^{+}=-\sum_{\lambda} \lambda|\lambda\rangle\langle\lambda|$ in terms of eigenfunctions $\hat{\Omega}^{+}|\lambda\rangle=\lambda|\lambda\rangle$ shows that an autocorrelation function can be represented as

$$
C(t)=\sum_{\lambda}|\langle A \mid \lambda\rangle|^{2} \exp (-\lambda t), \quad \text { for } t>0
$$

From this representation one immediately infers that $C(t)$ is completely monotone. By the famous Bernstein theorem [47] the converse is also true, i.e., any completely monotone function allows for a representation as a superposition of relaxing exponentials with positive weights.

For the VACF one concludes

$$
\left\langle\mathbf{v}(t) \cdot \mathbf{v}\left(t^{\prime}\right)\right\rangle=-\sum_{\lambda}\left|\left\langle\lambda \mid \hat{\Omega}^{+} \mathbf{r}\right\rangle\right|^{2} \exp (-\lambda t) .
$$

Since the equilibrium state $|0\rangle$ is annihilated by the backward Smoluchowski operator $\hat{\Omega}^{+}|0\rangle=$ 0 , the sum extends in fact only over positive eigenvalues $\lambda>0$.

Integration yields the time dependent diffusion coefficient

$$
D(t)=D_{0}-\sum_{\lambda>0}\left|\left\langle\lambda \mid \hat{\Omega}^{+} \mathbf{r}\right\rangle\right|^{2} \frac{1-\exp (-\lambda t)}{\lambda d}
$$

and one immediately infers that $D(t)$ is monotonically decreasing to the long-time diffusion coefficient

$$
D=D_{0}-\sum_{\lambda>0}\left|\left\langle\lambda \mid \hat{\Omega}^{+} \mathbf{r}\right\rangle\right|^{2} \frac{1}{\lambda d} .
$$

For the mean-square displacement one obtains the representation

$$
\delta r^{2}(t)=2 d D_{0} t-2 \sum_{\lambda>0}\left|\left\langle\lambda \mid \hat{\Omega}^{+} \mathbf{r}\right\rangle\right|^{2} \frac{\lambda t-1+\exp (-\lambda t)}{\lambda^{2}},
$$

valid for $t \geq 0$.

\section{References}

1. M. Sahimi, Heterogeneous Materials, vol. 22 of Interdisciplinary Applied Mathematics (Springer, New York, 2003).

2. G. Dagan, J. Fluid Mech. 145, 151 (1984). 
3. A. Meyer, J. Horbach, W. Kob, F. Kargl, and H. Schober, Phys. Rev. Lett. 93, 027801 (2004)

4. F. Kargl, A. Meyer, M. M. Koza, and H. Schober, Phys. Rev. B 74, 014304 (2006).

5. T. Voigtmann and J. Horbach, Europhys. Lett. 74, 459 (2006).

6. A. J. Moreno and J. Colmenero, J. Chem. Phys. 125, 164507 (2006).

7. A. J. Moreno and J. Colmenero, Phys. Rev. E 74, 021409 (2006).

8. N. Kikuchi and J. Horbach, EPL 77, 2600 (2007).

9. R. J. Ellis, Trends in Biochemical Sciences 26, 597 (2001), ISSN 0968-0004.

10. R. J. Ellis and A. P. Minton, Nature 425, 27 (2003), ISSN 0028-0836.

11. I. M. Tolić-Nørrelykke, E.-L. Munteanu, G. Thon, L. Oddershede, and K. Berg-Sørensen, Phys. Rev. Lett. 93, 078102 (2004).

12. I. Golding and E. C. Cox, Phys. Rev. Lett. 96, 098102 (2006).

13. M. Deverall, E. Gindl, E.-K. Sinner, H. Besir, J. Ruehe, M. Saxton, and C. Naumann, Biohpys. J. 88, 1875 (2005).

14. A. Kusumi, C. Nakada, K. Ritchie, K. Murase, K. Suzuki, H. Murakoshi, R. S. Kasai, J. Kondo, and T. Fujiwara, Annu. Rev. Biophys. Biomol. Struct. 34, 351 (2005).

15. M. Weiss, H. Hashimoto, and T. Nilsson, Biophys. J. 84, 4043 (2003), ISSN 0006-3495.

16. M. R. Horton, F. Höfling, J. O. Rädler, and T. Franosch, submitted to Soft Matter (2009).

17. H. van Beijeren, Rev. Mod. Phys. 54, 195 (1982).

18. D. Stauffer and A. Aharony, Introduction to Percolation Theory (Taylor \& Francis, London, 1994), 2nd ed.

19. F. Höfling, T. Franosch, and E. Frey, Phys. Rev. Lett. 96, 165901 (2006).

20. F. Höfling, T. Munk, E. Frey, and T. Franosch, J. Chem. Phys. 128, 164517 (2008).

21. F. Höfling, Ph.D. thesis, Ludwig-Maximilians-Universität München (2006), ISBN 978-386582-426-4.

22. B. J. Sung and A. Yethiraj, Phys. Rev. Lett. 96, 228103 (2006).

23. B. J. Sung and A. Yethiraj, J. Phys. Chem. B 112, 143 (2008), ISSN 1520-6106.

24. B. J. Sung and A. Yethiraj, J. Chem. Phys. 128, 054702 (2008).

25. C. Bruin, Phys. Rev. Lett. 29, 1670 (1972).

26. C. Bruin, Physica 72, 261 (1974).

27. B. J. Alder and W. E. Alley, J. Stat. Phys. 19, 341 (1978).

28. B. J. Alder and W. E. Alley, Physica A 121, 523 (1983).

29. W. E. Alley, Ph.D. thesis, California Univ., Davis (1979).

30. C. P. Lowe and A. J. Masters, Physica A 195, 149 (1993).

31. A. Weijland and J. M. J. van Leeuwen, Physica (Amsterdam) 38, 35 (1968).

32. M. H. Ernst and A. Weijland, Phys. Lett. A 34, 39 (1971).

33. F. Höfling and T. Franosch, Phys. Rev. Lett. 98, 140601 (2007).

34. J. Machta and S. M. Moore, Phys. Rev. A 32, 3164 (1985).

35. D. ben Avraham and S. Havlin, Diffusion and Reactions in Fractals and Disordered Systems (Cambridge University Press, Cambridge, 2000).

36. M. P. M. den Nijs, J. Phys. A 12, 1857 (1979).

37. B. Nienhuis, Phys. Rev. Lett. 49, 1062 (1982).

38. P. Grassberger, Physica A 262, 251 (1999).

39. A. Scala, T. Voigtmann, and C. De Michele, J. Chem. Phys. 126, 134109 (2007).

40. J. Quintanilla, S. Torquato, and R. M. Ziff, J. Phys. A 33, L399 (2000).

41. J. A. Quintanilla and R. M. Ziff, Phys. Rev. E 76, 051115 (2007).

42. P. H. Colberg and F. Höfling, Accelerating glassy dynamics on graphics processing units (2009), arXiv:0912.3824 [cond-mat.soft].

43. T. Franosch, F. Höfling, T. Bauer, and E. Frey, submitted to Chem. Phys. (2010).

44. A. Kammerer, F. Höfling, and T. Franosch, EPL 84, 66002 (2008).

45. L. Santen and W. Krauth, Nature (2000).

46. M. Bayer, J. M. Brader, F. Ebert, M. Fuchs, E. Lange, G. Maret, R. Schilling, M. Sperl, and J. P. Wittmer, Phys. Rev. E 76, 011508 (2007).

47. W. Feller, An Introduction to Probability Theory and Its Applications (Wiley, 1968), ISBN 0471257087. 